Case Report

\title{
SPIRAL CT A SEARCH ENGINE FOR DIAGNOSING MULTIPLE CANALS - A RARE CASE REPORT
}

\author{
Kiran Halkai ${ }^{1}$, Rahul Halkai ${ }^{2}$, Mithra N. Hegde ${ }^{3}$, Sapna S. ${ }^{4} \&$ Narender Reddy M. ${ }^{5}$ \\ ${ }^{1}$ Senior Lecturer, Department of Conservative \& Endodontics, S.N. Dental College, Gulbarg, ${ }^{2} P h . D$. Student, \\ ${ }^{3}$ HOD \& Senior Professor, Department of Conservative \& Endodontics, A.B. Shetty M emorial Institute of Dental \\ Sciences, Nitte University, M angalore - $575018,{ }^{4}$ Senior lecture, Department of Conservative \& Endodontics, \\ YMT Dental College \& Hospital, Navimumbai, ${ }^{5}$ SVS Dental College, M ahaboobnagar, Andhra Pradesh, INDIA.

\section{Correspondence:} \\ Kiran Halkai \\ Senior Lecture, Department of Conservative \& Endodontics, S.N. Dental College. Gulbarga, Karnataka, India. \\ Mobile : +91 7204166552 E-mail : drkiranc_15@yahoo.com

\begin{abstract}
:
Successful treatment of any case mainly depends on proper diagnosis; this is particularly true when we are doing an endodontic treatment. Internal anatomy of root canal system varies a lot and predisposes to inadequate root canal preparation and should be recognized before or during treatment. Proper knowledge of these anatomical variations and proper diagnosis are of paramost important. As the routine diagnostic aids, radiograph is a two dimensional representation of a three dimensional object where the internal anatomy of the tooth cannot be appreciated properly. Spiral computed tomography (SCT) has got an advantage as it gives a 3 dimensional image of a given object. We present an interesting case of multiple canals in all the teeth except incisors in a single patient which was diagnosed with the aid of Dental CT and its potential role, this diagnostic method can help in the field of endodontics
\end{abstract}

Keywords: multiple canals, Spiral computed tomography.

\section{Introduction:}

Success of root canal treatment mainly depends on proper cleaning, shaping and filling three dimensionally, it all depends on thorough knowledge of both external and internal anatomy of the root canal system ${ }^{1}$, this is crucial, because of root canal morphological variation, morphological variation is a constant challenge for proper diagnosis and success of endodontic therapy. Extra roots and root canals if not detected and if not obturated completely can lead to endodontic failure ${ }^{2}$ It is common to see extra canals in few particular teeth ${ }^{3,}{ }^{4}$ but it is uncommon to find extra canals in multiple teeth in a single patient. As we enter this new millennium and its accompanying realm of technologic advances it is evident

\begin{tabular}{|c|}
\hline Access this article online \\
\hline Quick Response Code \\
\hline \\
\hline
\end{tabular}
that dental surgeons should utilize modern diagnostic tools for successful treatment. Diagnostic imaging and technology helps to develop and implement a cohesive and comprehensive treatment plan for patients. conventional intraoral periapical radiographs are routinely used to assess the root canal anatomy, but owing to their inherent limitations as it is a two dimensional representation of a three dimensional object, they are not very helpful in assessing complex root canal anatomy. The ideal method of precise determination of the root canal morphology of a tooth is serial sectioning of the tooth, which is impractical in clinical situations. Therefore, diagnostic methods like spiral computed tomography (SCT) are useful in such conditions. The technique of dental CT, also called Dentascan, was developed by Schwarz et al. ${ }^{5}$ in 1987 primarily utilized for evaluating the teeth, the jaws and surrounding hard tissue morphology, pre-evaluation of implant sites, buccolingual extend of cysts, tumors, periapical lesions. Which creates images in three dimensions. ${ }^{3,4}$ Role of Dental CT in endodontics is still under emerging stages. This article highlights the diagnostic role of spiral CT in assessment of variations of root canal morphology and thus helpful in predicting the prognosis of a complex case. 


\section{Case Report :}

A 25 year male patient reported to department of conservative dentistry $\&$ endodontics, with a chief complaint of intermittent pain in upper left back region since 2 months which has increased in intensity for the past 2 days. The patient's medical history was non contributory. Medical and dental history was not significant. Oral findings revealed carious maxillary left first molar (pre operative radiograph) (Fig1) Local anaesthesia was administered and under strict asepsis, with placement of rubber dam, access cavity preparation was done. We found some morphological variation, after through searching 5 canals were located, Intra oral periapical radiograph was taken with $\mathrm{K}$ files in place for working length determination and it revealed the presence of five canals(Fig2). To study the variation in root canal anatomy dental CT was planned. An informed consent was obtained from the patient, SCT scan was performed with high resolution multi detector CT scanner (Somaton Sensation 64, Siemens, Germany) (16 slices/second), as per recommendations given by

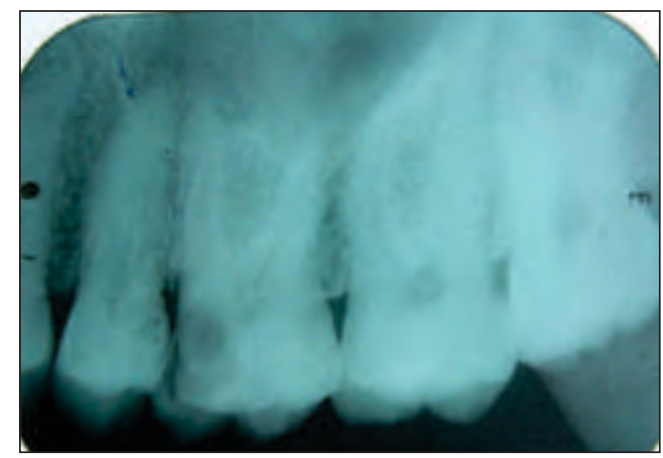

Fig 1: Pre operative radiograph.

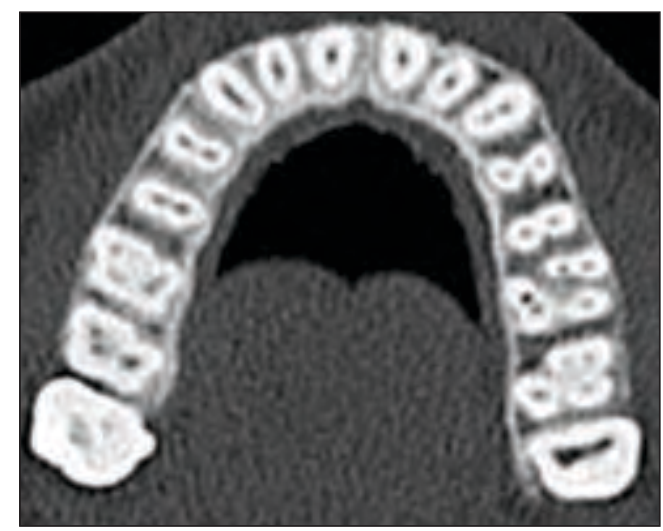

Fig 3: Axial view of maxillary arch at apical one third of all the teeth, showing extra canals in all the teeth except incisors.
Christoph et $\mathrm{al}^{12}$. To reduce the radiation dosage (collimation, $1 \mathrm{~mm}$; pitch, 2; tube voltage, $80 \mathrm{kV}$; tube current, $40 \mathrm{~mA}$ ). The scan reconstruction interval was 0.8 $\mathrm{mm}$, resulting in 60-100 overlapping images. Axial images were transmitted to a commercially available dental program (Denta scan, Advantage Windows; General Electric, Buc, France) to reformat panoramic and crosssectional images in all 3 planes at different levels to determine the canal morphology. Spiral CT scan slices revealed five canals (two mesio buccal, two palatal, and one distobuccal) (Fig3) in the left maxillary first molar. Two separate canals in both palatal and mesial roots i.e., mesio palatal and disto Palatal (MP and DP) and MB1, M B2 and one distal canals, both palatal and mesial canals had 2 separate canal exits confirmed by SCT(Fig 3).

One more important finding was multiple extra canals in all the teeth except incisors confirmed by CT scan (Fig 3) . Four canals in right and left maxillary second molar, 4 canals in right maxillary first molar, 2 canals in maxillary

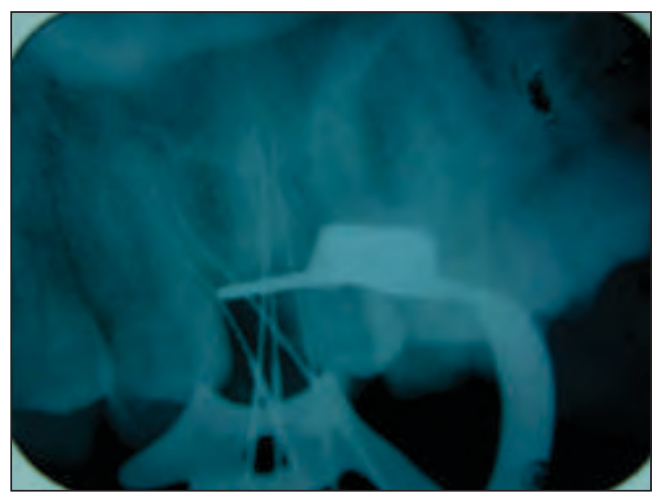

Fig2: Working length determination.

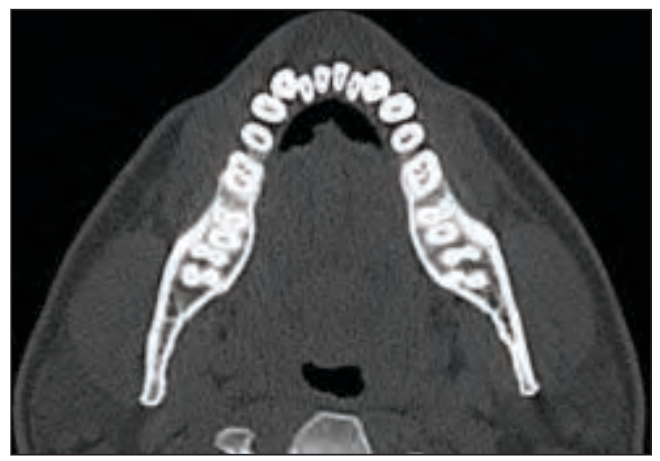

Fig 4 : Axial view of mandibular arch at apical one third of all the teeth 
and mandibular right \& left first and second premolars. 2 canals in right $\&$ left maxillary and mandibular canines and four canals in right and left mandibular first and third molars(Fig 3,4,). Paraxial images revealed periapical pathology in all the three roots of left maxillary first molar with periodontal ligament widening in palatal and mesiobuccal root.

\section{Discussion :}

The most frequent cause of endodontic failure is the apical percolation and subsequent diffusion into the canal. The main reasons for this failure are incomplete canal obturation or an untreated canal, most common reason is undetected canal as it goes unnoticed. ${ }^{6}$ Weine et al ${ }^{7}$ and vertucci ${ }^{8}$ provided the clinical classification of variations in the root canal system and stressed the importance of familiarity with the root canal morphology before starting the endodontic treatment. Therefore proper identification of the canals and further endodontic management are essential for successful endodontic treatment. Extra canals are a common findings and missing of these canals leads to endodontic treatment failure. The majority of endodontic literature describes the maxillary first molar as having 3 roots with 3 or 4 root canals. The prevalence of maxillary first molars with 2 palatal canals is rare. Also literature is scarce regarding presence of 2 separate palatal canals with separate orifices/ separate exits or single exit. ${ }^{9,10,11}$. Another in vivo study found two canals in the mesiobuccal roots of maxillary first molars $77 \%$ of the time, and, of these, $62 \%$ had two apical foramina. The incidence of accessory canals in the furcation of maxillary molars, canals that extend all the way from the pulpal floor to the furcation area is $48 \%$ in one study and $68 \%$ in another ${ }^{12,}{ }^{13}$. In mandibular molars, through-and-through furcal accessory canals are found $56 \%$ of the time in one study and $48 \%$ in another 7.8 . Mandibular molars also exhibit extra root canals, apart from normal 3 canals. Although as many as five canals ${ }^{14}$ and as few as one and two canals rarely occur in mandibular molars, four canals are not unusual ${ }^{15,16}$. Premolar teeth are also prone to have additional canals. Maxillary first premolars, which generally have two canals, have three canals 5 to $6 \%$ of the time ${ }^{17}$. $24 \%$ of maxillary second premolars have second root canals and occasionally three canals $^{18}$. Mandibular premolars are notorious for having extra canals $26.5 \%$ in first premolars and $13.5 \%$ in second premolars ${ }^{19}$. M axillary and mandibular canines usually has a single canal, only $3 \%$ of canines have 2 canals which usually join (75\%) in the apical third and exit through a single foramen) which is a rarest occurrence. Radiographic examination is an essential component of the management of endodontic problems. The amount of information gained from conventional radiographs and digitally captured periapical radiographs is limited by the fact that the three-dimensional anatomy of the area being radiographed is compressed into a two-dimensional image $^{20}$. Hence, anatomy in the third dimension cannot be assessed on radiographs. Because root canals tend to lie one behind the other in buccolingual plane, they get superimposed onto each other on periapical panoramic radiographs and easily go undetected ${ }^{1}$. Newer diagnostic methods such as Spiral Computed tomography (SCT) greatly facilitate to access the internal root canal morphology. One distinct advantage of SCT scanning over the conventional radiograph is that it allows the operator to look at multiple slices of tooth roots and their root canal systems. Robinson et al ${ }^{21}$ reported that CT images identified a greater number of morphologic variations than panoramic radiographs.

The use of spiral computerized tomography (SCT) scans in dentistry has increased dramatically in the past 2 decades. SCT scans acquire raw projection data with a spiralsampling locus in a relatively short period. Without additional scanning time, these data can be viewed as conventional transaxial images such as multiplanar reconstructions or as three-dimensional reconstructions. With SCT scans, it is possible to reconstruct overlapping structures at arbitrary intervals; therefore the ability to resolve small subjects is increased. It provides axial, panoramic, paraxial and $3^{\text {rd }}$ volume rendering which helps in diagnosis purpose. It is operator friendly software allowing the dentist to assess the images in dental office. They have drastically reduced scan time and effective dosages. ${ }^{22,23,24}$. The only disadvantage with the spiral CT 
technique can be its cost effectiveness for the patient. The CT scan would be relatively more expensive than a conventional radiograph for the patient. With further advancement in radiology and cone beam computed tomography evolving rapidly and providing three dimensional images at a less radiation dose, this could be the next diagnostic aid in endodontics may be surpassing the conventional tools.

The present paper, highlights the importance of Spiral CT scanning for a better understanding of the complex root canal anatomy. SCT confirmed the unusual anatomy of multiple root canals in multiple teeth in a single patient on

\section{References:}

1. Schwarz MS, Rothman SL, Rhodes ML, Chafetz N . Computed tomography.l. Preoperative assessment of the mandible for endosseous implant surgery. Int J Oral Maxillofac Implants 1987 2:137- 141.

2. DelBalso AM, Greiner FG, Licata M. Role of diagnostic imaging in evaluation of the dental implant patient. Radiographics 1994; 14:699719

3. James J. Abrahams: Dental CT Imaging: A Look at the Jaw: Radiology, 2001; 219:334-345.

4. John II, Van TH, Carl EH, Gerald N G, Thomas S, Paul AR, Endodontic cavity preparation. In Endodontics, Fifth edn. 2002 BC Decker Inc Pg 405-570.

5. European Society of Endodontology. Quality guidelines for endodontic treatment: consensus report of the European society of Endodntology. Int EndodJ 2006;39, 921-930

6. Weine FS, Healey HJ, Gerstein H, Evanson I. Canal configuration in the Mesiobuccal root of the maxillary first molar and its endodontic significance. Oral Surg OralM ed Oral Pathol 1969;28:419-425.

7. Vertucci F). Root canal anatomy of the human permanent teeth. Oral Surg OralM ed Oral Pathol 1984;58:589-599.

8. Wong M. Maxillary first molar with three palatal canals. J Endod 1991;17:298-299.

9. M aggiore F, Jou YT, Kim S. A six-canal maxillary first molar: case report. Int EndodJ 2002; 35:486-491.

10. Kiran Halkai, Rahul Halkai, Manisha Choudary, Endodontic management of maxillary first molar with two palatal canals and two mesiobuccal canals diagnosed with the aid of Spiral computed tomography - A case report. Endodontology, vol 25, issue 1,pgs 115120

11. Vertucci F), Anthony RL. A scanning electron microscopic investigation of accessary foramina in the furcation and pulp chamber floor of molar teeth. Oral Surg 1986; 62:319.

12. Niemann RW, et al. Dye ingress in molars: furcation to chamber floor. JOE 1993; 19:293.

13. Beatty RG, Krell K.M andibular molars with five canals: report of two cases. J Am Dent Assoc 1987; 114:802. routine examination. Literature survey is also scare for the study and occurrence of multiple canals in a single patient in multiple teeth ${ }^{25}$ presenting extra canals in all the teeth except the incisors.

\section{Conclusion :}

We presented an unusual case of multiple extra canals in all the teeth except in the incisors in a single patient which were detected on reformatting software. Dental CT assisted in formulating better treatment plan for this case. We recommend that Dental CT should be utilized in complicated cases as routine examination tool for better prognosis.

14. Walker RT. Root form and canal anatomy of mandibular second molars in a southern Chinese population. JOE 1988; 14:325.

15. Sierashi SM. Identification and endodontic management of threecanaled maxillary premolars. JOE 1989; 15:29.

16. Vertucci FJ, Selig A, Gillis R. Root canal morphology of the human maxillary second premolar. Oral Surg 1974;38:456

17. Zillich R, Dowson J. Root canal morphology of mandibular first and second premolars. Oral Surg 1973;36:783.

18. Bjorndal L, Carlsen O, Theusen G, Darvann T, Kreiborg S.External and internal macromorphology in 3D- reconstructed maxillary molars using computerized x-ray microtomography. IEJ 1999;32:3-9

19. Sert S, Bayirli GS. Evaluation of the root canal configurations of the mandibular and maxillary permanent teeth by gender in the Turkish population. JOE 2004;30: 391-398

20. Reddy YP, Karpagavinayagam K, Subbarao CV. Management of dens invaginatus diagnosed by spiral computed tomography: a case report. J Endod 2008; 34: 1138-1142.

21. Soraya Robinson, C. Czerny, A. Gahleitner, T. Bernhart and F. M Kainberger,Dental CT evaluation of mandibular first premolar root configurations and canal variations .Oral Surg Oral Med Oral Patho Oral Radiol Endod 2002; 93:328-332.

22. Tachibana H, Matsumoto K. Applicability of x-ray computerised tomography in endodontics. Endod Dent Traumatol 1990;6:16-20.

23. Kim S, Baek S The microscope and endodontics. Dent Clin N Am 2004:48: 11-18

24. Nakata K, Izumi M, Iwama A, Naito M, Inamoto K, Ariji E, Nakamura H. Utility of dental computed tomography (CT) in endodontic therapy. Part 2: Diagnostic imaging of periapical lesions of each root of multirooted teeth. Japanese Journal of Conservative Dentistry 2004 47:608-615.

25. jayaprakash patil, sushma jaju, prashant $\mathrm{p}$ jaju. Dental CT as a diagnostic aid in a case of multiple extra canals. Endodonto 23;1:8690. 